\title{
Changes in the Gut Microbiota are Associated with Hypertension, Hyperlipidemia, and Type 2 Diabetes Mellitus in Japanese Subjects
}

\author{
Tomohisa Takagi $^{1,2, * \mathbb{D}}$, Yuji Naito ${ }^{1} \mathbb{C}$, Saori Kashiwagi ${ }^{1}{ }^{\mathbb{D}}$, Kazuhiko Uchiyama ${ }^{1}$, \\ Katsura Mizushima ${ }^{1}$, Kazuhiro Kamada ${ }^{1}$, Takeshi Ishikawa ${ }^{1}$, Ryo Inoue ${ }^{3}{ }^{\circledR}$, Kayo Okuda ${ }^{4}$, \\ Yoshimasa Tsujimoto ${ }^{4}$, Hiromu Ohnogi ${ }^{4}$ and Yoshito Itoh ${ }^{1}$ \\ 1 Molecular Gastroenterology and Hepatology, Graduate School of Medical Science, \\ Kyoto Prefectural University of Medicine, Kyoto 602-8566, Japan; ynaito@koto.kpu-m.ac.jp (Y.N.); \\ skashiwa@koto.kpu-m.ac.jp (S.K.); k-uchi@koto.kpu-m.ac.jp (K.U.); mizusima@koto.kpu-m.ac.jp (K.M.); \\ k-kamada@koto.kpu-m.ac.jp (K.K.); iskw-t@koto.kpu-m.ac.jp (T.I.); yitoh@koto.kpu-m.ac.jp (Y.I.) \\ 2 Department for Medical Innovation and Translational Medical Science, Graduate School of Medical Science, \\ Kyoto Prefectural University of Medicine, Kyoto 602-8566, Japan \\ 3 Laboratory of Animal Science, Department of Applied Biological Sciences, Faculty of Agriculture, \\ Setsunan University, Osaka 573-0101, Japan; ryo.inoue@setsunan.ac.jp \\ 4 Contract Development \& Manufacturing Center 4, Takara Bio Inc., Shiga 525-0058, Japan; \\ okudakz@takara-bio.co.jp (K.O.); tsujimotoy@takara-bio.co.jp (Y.T.); onogih@takara-bio.co.jp (H.O.) \\ * Correspondence: takatomo@koto.kpu-m.ac.jp; Tel.: +81-75-251-5508
}

Received: 4 September 2020; Accepted: 27 September 2020; Published: 30 September 2020

\begin{abstract}
The human gut microbiota is involved in host health and disease development. Therefore, lifestyle-related diseases such as hypertension (HT), hyperlipidemia (HL), and type 2 diabetes mellitus (T2D) may alter the composition of gut microbiota. Here, we investigated gut microbiota changes related to these diseases and their coexistence. This study involved 239 Japanese subjects, including healthy controls (HC). The fecal microbiota was analyzed through the isolation of bacterial genomic DNA obtained from fecal samples. Although there were no significant differences in the microbial structure between groups, there was a significant difference in the $\alpha$-diversity between HC and the patients in whom two diseases coexisted. Moreover, Actinobacteria levels were significantly increased, whereas Bacteroidetes levels were significantly decreased in all disease groups. At the genus level, Bifidobacterium levels were significantly increased in the HL and T2D groups, as were those of Collinsella in all disease groups. In contrast, Alistipes levels were significantly lower in the HL group. Furthermore, metabolic enzyme families were significantly increased in all disease groups. Interestingly, the structure and function of the gut microbiota showed similar profiles in all the studied diseases. In conclusion, several changes in the structure of the gut microbiota are associated with T2D, HT, and HL in Japanese subjects.
\end{abstract}

Keywords: gut microbiota; hypertension; hyperlipidemia; type 2 diabetes mellitus

\section{Introduction}

Hypertension (HT), hyperlipidemia (HL), and type 2 diabetes mellitus (T2D) have become major public health issues throughout the world, and are important risk factors for cerebral and cardiovascular diseases in Japan, as well as in Western countries [1-4]. It has been reported that the prevalence of HT, $\mathrm{HL}$, and T2D in Japan is $15.2 \%, 6.9 \%$, and $4.8 \%$ [5], respectively. In addition, it is well known that these diseases are prone to happen as comorbid conditions. 
Growing clinical and animal research-based data surrounding the human gut microbiome have revealed the importance of the gut microbiome in homeostatic functions throughout the host body, and also that its composition is linked to host health and disease development [6]. Several studies have demonstrated that an altered composition of commensal bacteria is associated with the development of inflammatory bowel diseases and irritable bowel syndrome, as well as allergies and obesity in humans. Furthermore, gut microbiota dysbiosis has been recognized as having a key importance in lifestyle-related diseases.

Several studies have reported that individuals with HT, HL, and T2D, as well as those with comorbid conditions, have alterations in their intestinal microbiota profiles [7]. Qin et al. demonstrated that Chinese patients with T2D were characterized by a moderate degree of gut microbial dysbiosis, a decrease in the abundance of some universal butyrate-producing bacteria, and an increase in various opportunistic pathogens [8]. Moreover, the risk of developing T2D and HL has been reported to be correlated with an alteration in the composition and the function of the intestinal microbiota and the alleviation of hyperglycemia and hypercholesteremia by drug treatment, which shifts the gut microbiota structure, favoring beneficial bacteria such as Blautia and Faecalibacterium [9]. Regarding the gut microbiota in patients with HT, opportunistic pathogenic taxa such as Klebsiella, Streptococcus, and Parabacteroides merdae were widely distributed, whereas short-chain fatty acid producers such as Roseburia and Faecalibacterium prausnitzii were less abundant [10].

Nishijima et al. clearly demonstrated that the gut microbiome of the Japanese population is considerably different from that of other populations, and some studies have shown that compositional microbial changes in diseased Japanese subjects are linked to T2D [11]. Hashimoto et al. reported that the gut microbiota and its functional profiles in Japanese patients with T2D were significantly different from those in healthy individuals, and that sucrose intake was closely associated with these differences [12]. However, the detailed characteristics of gut microbiota in Japanese patients with HT, HL, T2D, and those with comorbid conditions remain unclear. In the present study, we investigated the profiles of gut microbiota in Japanese patients with these three diseases and found that several changes in the structure of the gut microbiota are associated with them.

\section{Materials and Methods}

\subsection{Study Population}

From November 2016 to April 2017, we prospectively selected 239 subjects from our outpatient clinic. Eligible subjects included male and female individuals aged between 20 and 90 years. HT was defined as systolic blood pressure $\geq 140 \mathrm{mmHg}$, diastolic blood pressure $\geq 90 \mathrm{mmHg}$, or current use of antihypertensive drugs. HL was defined as serum low-density lipoprotein cholesterol concentration $\geq 140 \mathrm{mg} / \mathrm{dL}$, high-density lipoprotein cholesterol concentration $<40 \mathrm{mg} / \mathrm{dL}$, triglyceride concentration $\geq 150 \mathrm{mg} / \mathrm{dL}$, or current use of agents against HL. T2D was defined as fasting plasma glucose level $\geq 126 \mathrm{mg} / \mathrm{dL}$, hemoglobin A1c level $\geq 6.5 \%$, or current use of antidiabetic drugs. Healthy controls (HC) did not have any gastrointestinal inflammatory diseases such as inflammatory bowel disease or functional gastrointestinal disorders such as functional dyspepsia and irritable bowel syndrome. Additional HC exclusion criteria included medication of antibiotics, corticosteroids, immunosuppressants, or acid-suppressing agents (proton pump inhibitors and histamine-type 2 receptor blockers) within the past three months, as well as a history of underlying malignant disease. In addition, patients with serious metabolic, respiratory, cardiologic, renal, hepatic, hematologic, neurologic, or psychiatric dysfunction and who regularly used medications that affect intestinal motility such as laxatives, antidepressants, opioid narcotic analgesics, anticholinergics, prokinetic agents, and prebiotics or probiotics were excluded. Subjects who were pregnant or lactating were also excluded. About a third of the subjects enrolled in this study were also involved with a previous study regarding the analysis of gut microbiota in healthy subjects and patients with T2D [12]. 
Overall, $54 \mathrm{HC}, 97$ patients with HT, 96 patients with HL, and 162 patients with T2D were enrolled in this study (Table 1). As shown in Figure 1, 72 patients had two of these diseases, namely, 36 patients had T2D and HL, 31 patients had T2D and HT, and 5 patients had HL and HT, and were classified as the RISK2 group. In addition, 49 patients had all three diseases and were classified as the RISK3 group, and 64 patients had only one disease (46 patients with T2D, 6 patients with HL, and 12 patients with HT) and were classified as the RISK1 group.

Table 1. Baseline characteristics of enrolled subjects in this study.

\begin{tabular}{cccccc}
\hline & ALL & Healthy Control & Hypertension & Hyperlipidemia & Type 2 Diabetes \\
\hline Number of samples & 239 & 54 & 97 & 96 & 162 \\
Male/Female & $113 / 126$ & $21 / 33$ & $49 / 48$ & $51 / 45$ & $79 / 83$ \\
Age (median) & $68(16-88)$ & $65.5(16-88)$ & $69(37-87)$ & $69(37-87)$ & $69(37-87)$ \\
Height (median) & $158.9(135.0-185.3)$ & $159.5(140.0-178.0)$ & $160.0(135.0-180.5)$ & $160.7(140.0-180.5)$ & $160.0(140.0-185.3)$ \\
Weight (median) & $54.80(33.10-128.60)$ & $53.75(33.10-85.90)$ & $61.50(35.65-128.60)$ & $62.60(39.0-128.60)$ & $61.40(39-128.60)$ \\
\hline
\end{tabular}
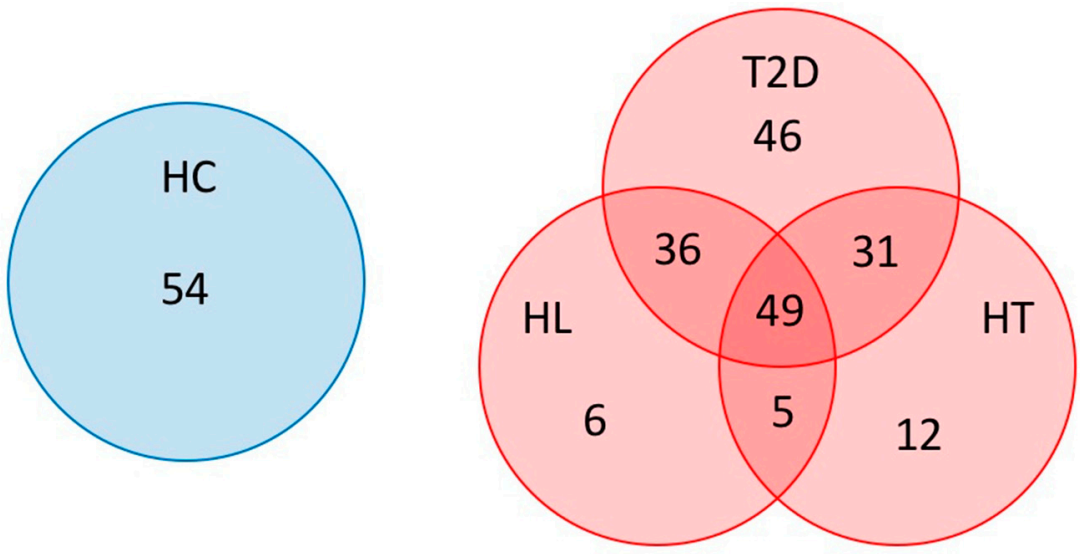

Figure 1. Subjects enrolled in this study. Patients overlapping T2D, HT, and HL were indicated using a Venn diagram. (HC: healthy controls, T2D: type 2 diabetes, HT: hypertension, HL: hyperlipidemia).

\subsection{Sample Collection and DNA Extraction}

Fecal samples were collected and gut bacterial composition analysis was performed according to previous reports [13-15]. Fecal samples the size of a grain of rice were collected using guanidine thiocyanate solution (Feces Collection kit; Techno Suruga Lab, Shizuoka, Japan). After vigorous mixing, the samples were stored at a temperature no higher than room temperature for a maximum of seven days until DNA extraction took place.

Genomic DNA was isolated using the NucleoSpin Microbial DNA Kit (Macherey-Nagel, Düren, Germany). Approximately $500 \mu \mathrm{L}$ of each stored fecal sample was placed in a microcentrifuge tube containing $100 \mu \mathrm{L}$ of Elution Buffer (EB). The mixture was then placed into a NucleoSpin Beads Tube with proteinase $\mathrm{K}$ and subjected to disruption with mechanical beads for $12 \mathrm{~min}$ at $30 \mathrm{~Hz}$ in the TissueLyser LT (Qiagen, Hilden, Germany). The subsequent extraction procedure was performed per the manufacturer's instructions. Extracted DNA samples were purified using the Agencourt AMPure XP (Beckman Coulter, Brea, CA, USA).

\subsection{Sequencing of the $16 S$ rRNA Gene}

Two-step polymerase chain reactions (PCRs) were performed in the purified DNA samples to obtain sequence libraries. The first PCR was performed for amplification and used a 16S (V3-V4) Metagenomic Library Construction Kit for NGS (Takara Bio Inc., Kusatsu, Japan) with primer pairs 341F (5'-TCGTCGGCAGCGTCAGATGTGTATAAGAGACAGCCTACGGGNGGCWGCAG-3') and 806R (5'-GTCTCGTGGGCTCGGAGATGTGTATAAGAGACAGGGACTACHVGGGTWTCTAAT-3'), corresponding to the V3-V4 region of the 16S rRNA gene. The second PCR was performed to add the index sequences for the Illumina sequencer with a barcode sequence using the Nextera XT Index 
kit (Illumina, San Diego, CA, USA). The prepared libraries were subjected to the sequencing of 250 paired-end bases using the MiSeq Reagent v3 kit and the MiSeq (Illumina) at the Biomedical Center at Takara Bio.

\subsection{Microbiome Analysis}

The processing of sequence data, including assembly, chimera check, operational taxonomic unit (OTU) definition, and taxonomy assignment, was performed using CD-HIT-OTU version 0.0 .1 and QIIME version 1.8.0 [16,17]. The taxonomy assignment of the resulting OTU was completed using RDP classifier version 2.2 and the Greengenes database [18,19]. Statistical differences $(p<0.05)$ in the relative abundance of bacterial phyla and genera among groups were evaluated using Wilcoxon rank-sum tests and the Benjamini-Hochberg method. These data have been deposited with links to BioProject accession number PRJDB10610 in the DDBJ BioProject database.

The $\alpha$-diversity indices (observed species (OTU richness estimation) and Shannon index (OTU evenness estimation)) were calculated by QIIME version 1.8.0 and were statistically analyzed using Student's unpaired $t$-tests. The $\beta$-diversity was estimated using the UniFrac metric to calculate the distances between the samples, and was visualized by principal coordinate analysis (PCoA).

Potential changes in the microbiome at the functional level were evaluated from sequence data downsampled to 10,000 reads using PICRUSt version 1.0.0, which uses 16S-rRNA sequence profiles to estimate metagenome content based on reference bacterial genomes and the Kyoto Encyclopedia of Genes and Genomes (KEGG) database [20,21]. The results were further statistically analyzed by Wilcoxon rank-sum tests and the Benjamini-Hochberg method. $p$-values $(<0.05)$ were used to determine statistically significant differences between the groups.

Statistical analysis was performed using $\mathrm{R}$ (version 3.4.3) and the final figures were generated with R package ggplot2 or Excel.

\subsection{Ethical Statements}

This study conformed to the code of ethics stated in the Declaration of Helsinki. The Ethics Committee of the Kyoto Prefectural University of Medicine approved the research protocol (permission no. ERB-C-534-6) and all participants provided written informed consent before enrollment. The study was registered at the University Hospital Medical Information Network Center (UMIN000019486).

\section{Results}

\subsection{Microbiota Diversity in Patients with HT, HL, and T2D}

Initially, we evaluated the diversity of gut microbiota using different $\alpha$-diversity indices (the observed species (OTU richness estimation) and the Shannon index (OTU evenness estimation)). They showed no statistically significant differences between $\mathrm{HC}$ and the patients with each disease (Figure 2A,B), though both indices showed statistically significant differences between $\mathrm{HC}$ and the RISK2 group. Subsequently, the overall structure of the gut microbiome for HC and the patients with these diseases using $\beta$-diversity indices was calculated for unweighted and weighted UniFrac distances (Figure 3). PCoA revealed that there were no microbial structural differences between HC and the patients with these diseases in unweighted and weighted distances using the two principal components (PC1 and PC2). 
(A)

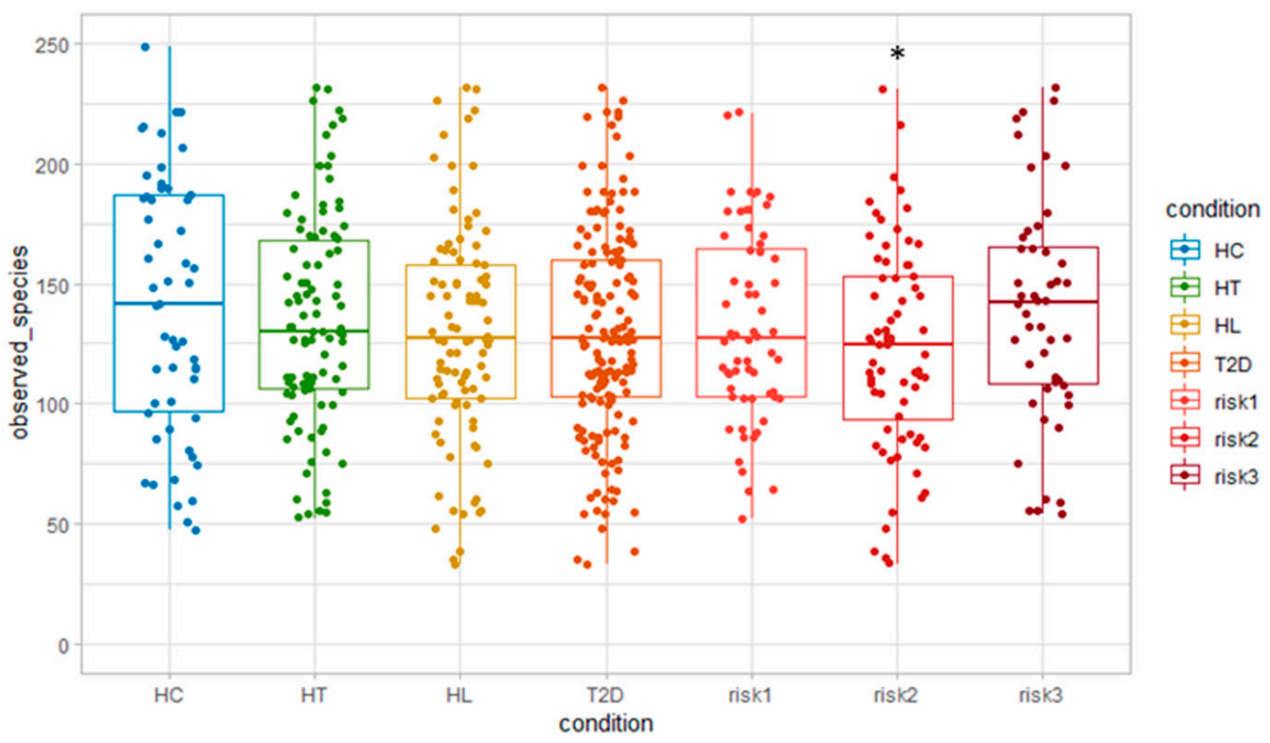

(B)

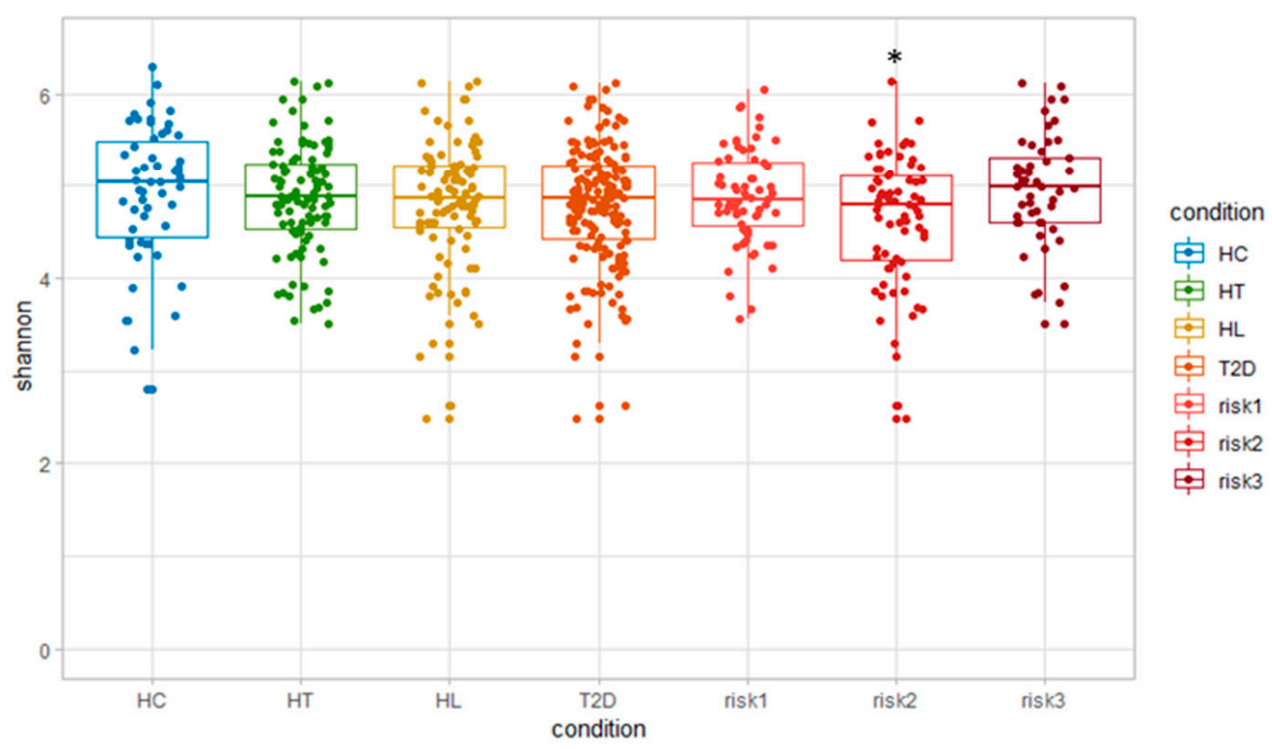

Figure 2. Analysis of $\alpha$-diversity in gut microbiota. We evaluated and compared $\alpha$-diversity indices: (A) observed species (operational taxonomic unit (OTU) richness estimation) and (B) Shannon index (OTU evenness estimation), using Student's unpaired $t$-tests ( ${ }^{*} p<0.05$ vs. HC). (HC: healthy controls, HT: hypertension, HL: hyperlipidemia, T2D: type 2 diabetes, RISK1: patients with only one disease among HT, HL, and T2D, RISK2: patients with two diseases among HT, HL, and T2D, RISK3: patients with the three diseases (HT, HL, and T2D)). 
(A)

(a)

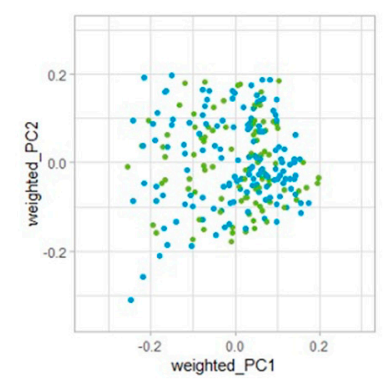

(B)

(a)

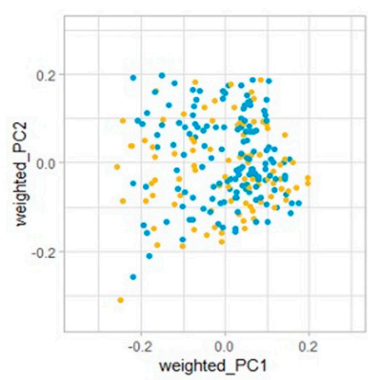

(C)

(a)

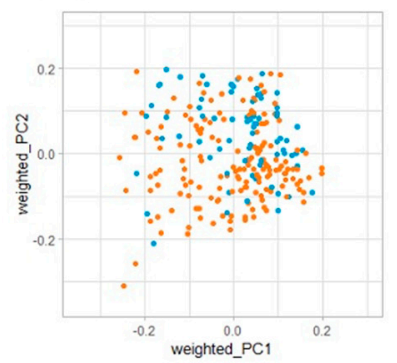

(D)

(a)

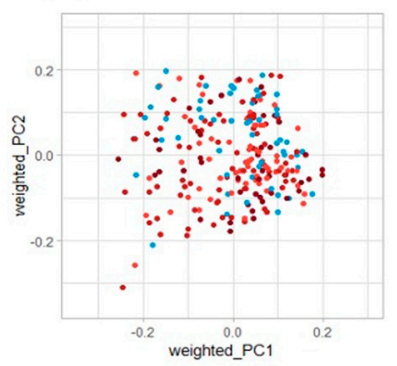

(b)

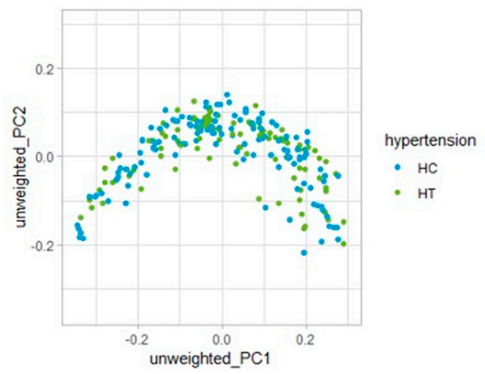

(b)

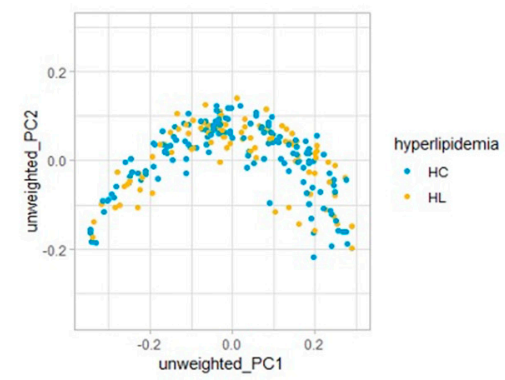

(b)

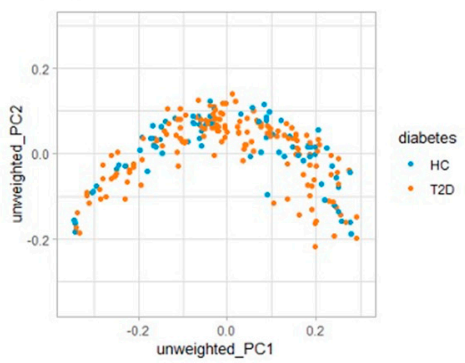

(b)

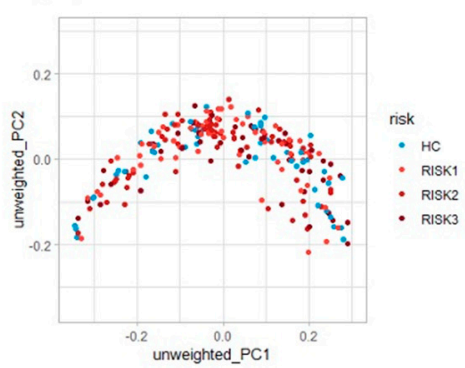

Figure 3. Principal coordinate analysis plots of gut microbiota. Distances were calculated with unweighted (a) and weighted (b) UniFrac for HT (A), HL (B), T2D (C), and RISK1, 2, and 3 (D) compared to HC. (HC: healthy controls, HT: hypertension, HL: hyperlipidemia, T2D: type 2 diabetes, RISK1: patients with only one disease among HT, HL, and T2D, RISK2: patients with two diseases among HT, HL, and T2D, RISK3: patients with the three diseases (HT, HL, and T2D)). 


\subsection{Microbiota Structure in Patients with T2D, HL, and HT}

The differences in the gut microbial structure in each group were taxonomically evaluated at the phylum level (Figure 4). In agreement with previous results, the microbiota composition included four predominant phyla: Firmicutes, Bacteroidetes, Actinobacteria, and Proteobacteria. Interestingly, the Actinobacteria phylum levels showed a statistically significant increase in all patient groups compared to HC. On the other hand, the Bacteroidetes phylum showed significantly decreased levels in all patient groups except in the RISK2 group compared to HC, although the abundance of Bacteroidetes phylum tended to decrease in the RISK2 group, as well as in other groups.

\section{(A)}

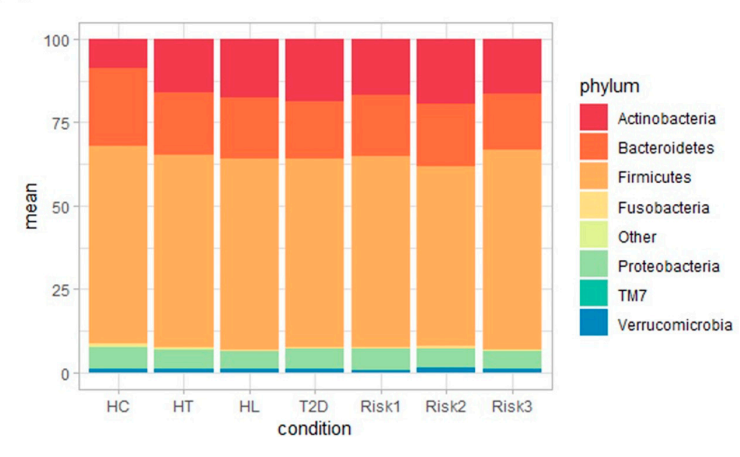

(B)

(a)

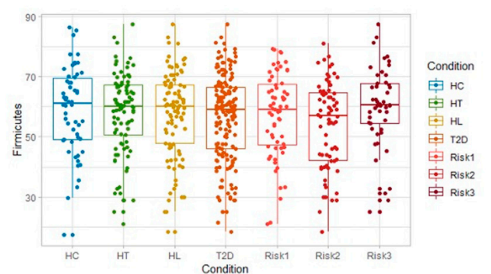

(c)

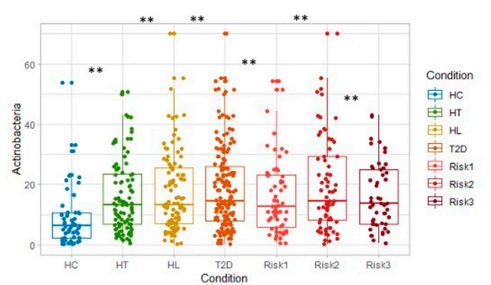

(e)

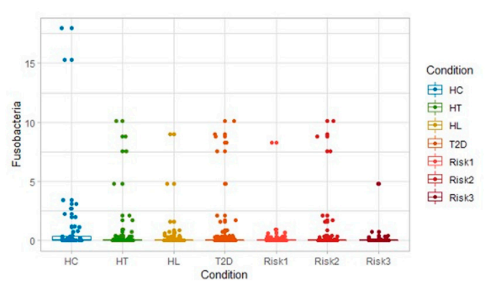

(b)

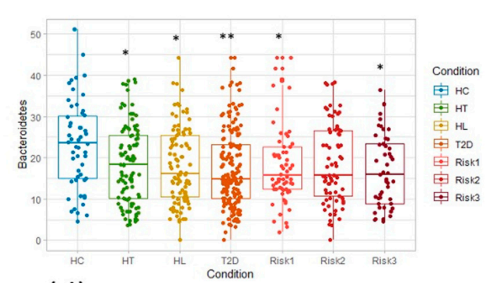

(d)

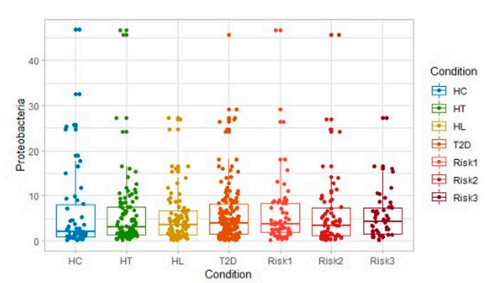

H. Comparative analysis of the taxonomic composition of the microbial community at the phylum level. Each component of the cumulative bar chart indicates a phylum (A). Each participant group was evaluated for Firmicutes (B-a), Bacteroidetes (B-b), Actinobacteria (B-c), Proteobacteria (B-d), and Fusobacteria (B-e) using Student's unpaired $t$-tests $\left({ }^{*} p<0.05,{ }^{* *} p<0.01\right.$ vs. HC). (HC: healthy controls, HT: hypertension, HL: hyperlipidemia, T2D: type 2 diabetes, RISK1: patients with only one disease among HT, HL, and T2D, RISK2: patients with two diseases among HT, HL, and T2D, RISK3: patients with the three diseases (HT, HL, and T2D)). 
Taxonomic changes in the microbial community were also evaluated at the genus level. As presented in Figure 5, the abundance of four genera showed significant differences between $\mathrm{HC}$ and the patients with the diseases. The levels of the Bifidobacterium genus were significantly increased in the HL, T2D, RISK1, and RISK2 groups compared to HC, and those of the Collinsella genus were also significantly increased in the HT, HL, T2D, RISK2, and RISK3 groups compared to HC, whereas the Escherichia genus had its levels significantly increased only in the RISK3 group. In contrast, the levels of the Alistipes genus were significantly decreased in the HL group compared to HC. Interestingly, these alterations tended to be similar in all groups.

(A)

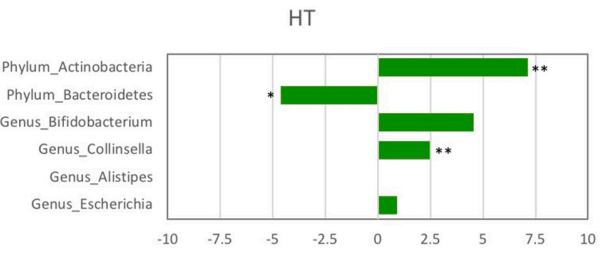

(B)

$\mathrm{HL}$

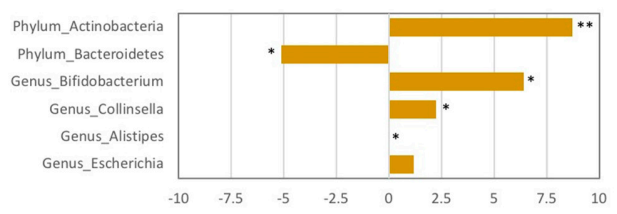

(C)

T2D

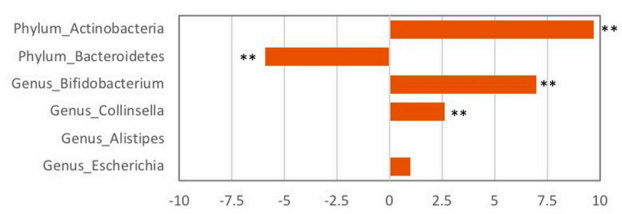

(D)

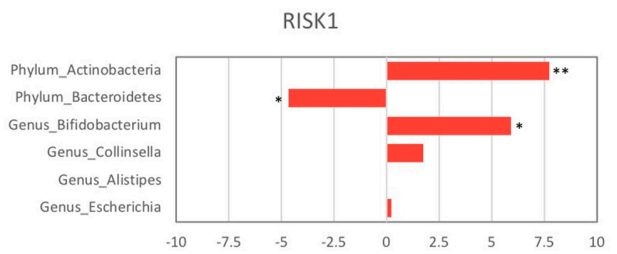

(E)

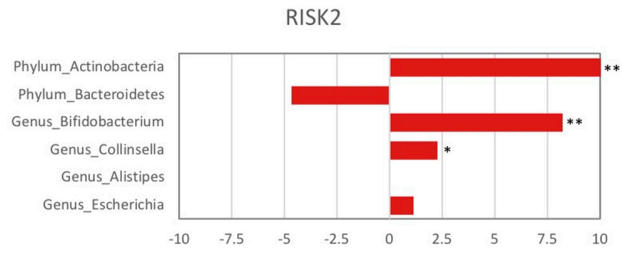

(F)

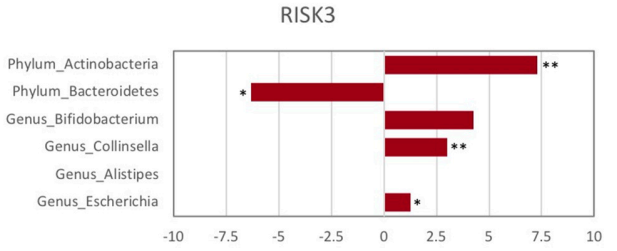

Figure 5. Relative abundance of gut microbiota at the genus level. The genera with significant differences among each group compared to HC are presented for HT (A), HL (B), T2D (C), RISK1 (D), RISK2 (E), and RISK3 (F), using Student's unpaired $t$-tests $\left({ }^{*} p<0.05,{ }^{* *} p<0.01\right.$ vs. HC). (HC: healthy controls, HT: hypertension, HL: hyperlipidemia, T2D: type 2 diabetes, RISK1: patients with only one disease among HT, HL, and T2D, RISK2: patients with two diseases among HT, HL, and T2D, RISK3: patients with the three diseases (HT, HL, and T2D)). 
Finally, we evaluated potential differences in the function of the microbiome using the PICRUSt software (Figure 6). Microbiome functions showed a similar pattern among patients with HT, HL, and T2D. When comparing these disease groups with HC at the second level of KEGG, the proportion of genes responsible for membrane transport and metabolic enzyme families was significantly increased in the HL and T2D groups, and in the HT, HL, and T2D groups, respectively, compared to HC.

(A)

HT

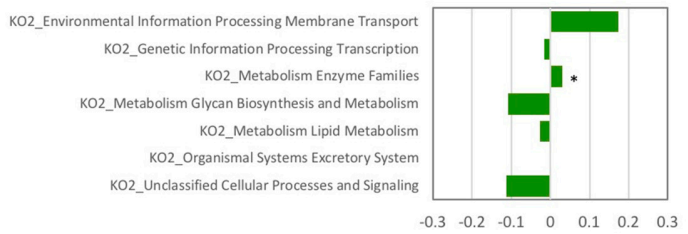

(B)

$\mathrm{HL}$

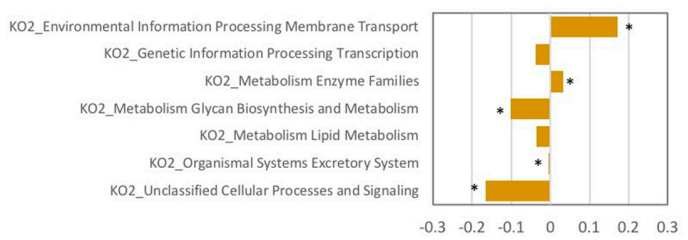

(C)

T2D

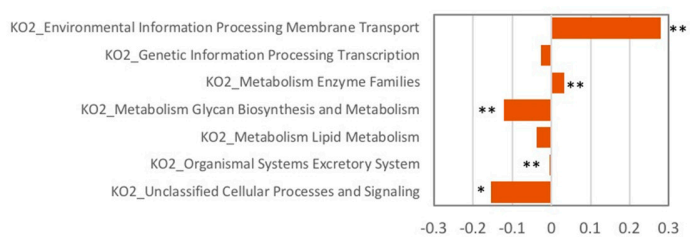

(D)

RISK1

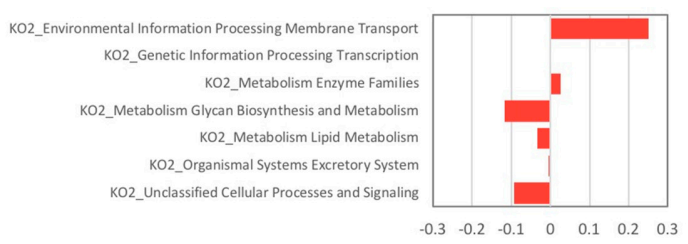

(E) RISK2

(F)
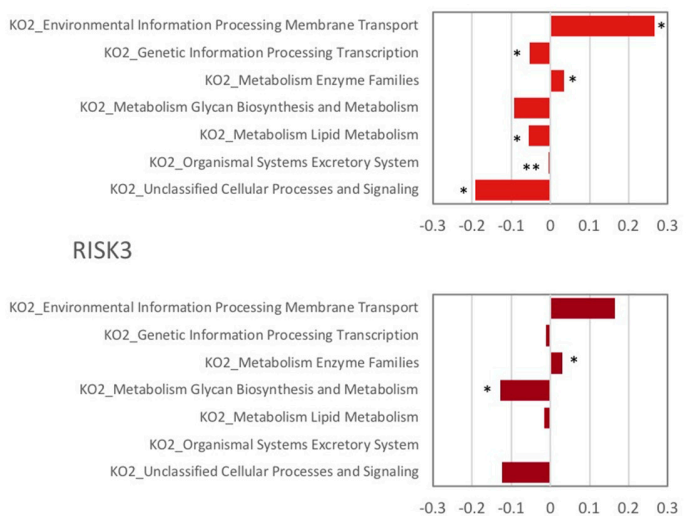

Figure 6. Relative abundance of functional pathways. The KEGG (Kyoto Encyclopedia of Genes and Genomes) database functional categories at the second level of KEGG and metabolic enzyme families are shown in the histograms for HT (A), HL (B), T2D (C), RISK1 (D), RISK2 (E), and RISK3 (F) compared to HC, using Student's unpaired $t$-tests $\left({ }^{*} p<0.05,{ }^{* *} p<0.01\right.$ vs. HC). (HC: healthy controls, HT: hypertension, HL: hyperlipidemia, T2D: type 2 diabetes, RISK1: patients with only one disease among HT, HL, and T2D, RISK2: patients with two diseases among HT, HL, and T2D, RISK3: patients with the three diseases (HT, HL, and T2D)). 


\section{Discussion}

In the present study, we analyzed the fecal gut microbiota in Japanese subjects with HT, HL, and T2D. Interestingly, these three diseases showed similar profiles of gut microbiota structure and functions. In addition, as lifestyle-related diseases, they often overlap, and such cases also showed similar gut microbiota profiles in our study. Importantly, the results of our functional analysis also presented a similar trend between HT, HL, and T2D.

The levels of the Actinobacteria phylum were significantly increased in patients with HT, HL, and T2D, and this increase was reflected in the increased abundance of the Bifidobacterium genus. Similarly, for comorbid conditions, the levels of the Actinobacteria phylum and Bifidobacterium genus were also significantly increased. Conversely, the abundance of the Bacteroidetes phylum was significantly decreased in patients with HT, HL, T2D, and comorbid conditions. In agreement with our results, Adachi et al. demonstrated that T2D patients had larger and smaller fecal populations of Bifidobacterium and Bacteroides, respectively, than the control individuals [22]. Interestingly, they also demonstrated that T2D patients had consumed more carbohydrates and had lower fecal propionate and butyrate concentrations, and that the levels of Bifidobacterium were negatively correlated with the carbohydrate intake. Hashimoto et al. also found that the Actinobacteria phylum was highly abundant in patients with T2D, whereas the Bacteroidetes phylum was less abundant [12]. In addition, the levels of diabetic-type gut microbes were altered by sucrose intake at the genus level, specifically Bacteroides and Bifidobacterium [12]. Therefore, a rich abundance of the Actinobacteria phylum and a lower abundance of the Bacteroidetes phylum seem to be characteristic of the gut microbiota of Japanese patients with T2D.

In the present study, the abundance of the Collinsella genus was also significantly increased in the HT, HL, T2D, RISK2, and RISK3 groups compared to HC. It has been reported that the Collinsella genus, which belongs to the Actinobacteria phylum, is associated with serum cholesterol and triglyceride levels [23]. Although the precise molecular mechanisms by which Collinsella affects host metabolism are unknown, its high abundance has been associated with obesity and T2D [24,25]. Interestingly, low dietary fiber might enable the overgrowth of Collinsella, and a structured weight loss program could significantly decrease Collinsella levels in patients with obesity and T2D [26,27].

In contrast, the abundance of the Alistipes genus was significantly decreased in the HL group compared to HC. The abundance of Alistipes, which belongs to the Bacteroidetes phylum, was lower in rodent models with high fat diet-induced hyperlipidemia $[28,29]$. In addition, the levels of this genus were also significantly decreased in obese Mexican individuals [30]. Meanwhile, they were positively correlated with systolic and diastolic blood pressure in Chinese hypertensive patients with treatment-naive hypertension [31].

This study has several limitations. The clinical investigation was performed with a limited number of subjects in a single center, and the analysis was performed on both patients being treated for HT, $\mathrm{HL}$, and T2D, and treatment-naive patients. This limitation may have affected the results because patients with these diseases often improve their dietary behaviors and control their intake of salt, sugar, and oil, and some therapeutic agents for these conditions, such as metformin, affect the structure of gut microbiota [5]. In addition, as we did not obtain the daily data of dietary intakes in this investigation, we could not analyze the association between the gut microbiota and dietary intakes. A detailed evaluation of the gut microbiota in a larger number of patients with these lifestyle-related diseases is needed in the near future.

\section{Conclusions}

In conclusion, this study demonstrated that several compositional changes in the gut microbiota are associated with lifestyle-related diseases such as HT, HL, and T2D, and that the gut environment seems to share a common pattern in these three diseases. Our data also highlight the crucial importance of gut microbiota through a better understanding of intestinal functions, and that patients with HT, $\mathrm{HL}$, and T2D have gut dysbiosis that may consequently contribute to disease onset and influence its 
clinical prognosis. Furthermore, homeostatic disturbances in the gut-related metabolism may underlie the pathogenesis of these diseases.

Author Contributions: Conceptualization, T.T., Y.N., and R.I.; methodology, T.T., Y.N., and R.I.; validation, S.K., K.M., T.I., and R.I.; formal analysis, K.O.; investigation, T.T., S.K., K.U., and K.K.; resources, K.O., Y.T., and H.O.; data curation, K.O.; writing —original draft preparation, T.T.; writing—review and editing, R.I., K.O., and Y.N.; supervision, Y.I.; project administration, K.U.; funding acquisition, Y.N. All authors have read and agreed to the published version of the manuscript.

Funding: This research was supported by the NARO Bio-oriented Technology Research Advancement Institution (R\&D matching funds on the field for Knowledge Integration and innovation), grant number 16824414.

Acknowledgments: We thank all members of the Department of Molecular Gastroenterology and Hepatology, Kyoto Prefectural University of Medicine Graduate School of Medical Science, for helping with this study.

Conflicts of Interest: Authors K.O., Y.T., and H.O. were employed by Takara Bio Inc. T.T. received lecture fees from Mochida Pharm. Co., Ltd. and Janssen Pharma K.K. Y.N. received scholarship funds from EA Pharma. Co., Ltd. and has been paid lecture fees by Janssen Pharma K.K., Mylan EPD Co., Takeda Pharma Co., Ltd., Mochida Pharm. Co., Ltd., EA Pharma Co., Ltd., Otsuka Pharma Co., Ltd., and Astellas Pharma Co., Ltd. Y.I. is affiliated with a department funded by donations from Nichinichi Pharmaceutical Co., Ltd. All other authors have no conflict of interest to declare.

\section{References}

1. Iwashita, M.; Matsushita, Y.; Sasaki, J.; Arakawa, K.; Kono, S. Relation of serum total cholesterol and other factors to risk of cerebral infarction in Japanese men with hypercholesterolemia. Circ. J. 2005, 69, 1-6. [CrossRef]

2. Wada, S.; Koga, M.; Toyoda, K.; Minematsu, K.; Yasaka, M.; Nagai, Y.; Aoki, S.; Nezu, T.; Hosomi, N.; Kagimura, T.; et al. Factors Associated with Intima-Media Complex Thickness of the Common Carotid Artery in Japanese Noncardioembolic Stroke Patients with Hyperlipidemia: The J-STARS Echo Study. J. Atheroscler. Thromb. 2018, 25, 359-373. [CrossRef]

3. Wake, M.; Oh, A.; Onishi, Y.; Guelfucci, F.; Shimasaki, Y.; Teramoto, T. Adherence and persistence to hyperlipidemia medications in patients with atherosclerotic cardiovascular disease and those with diabetes mellitus based on administrative claims data in Japan. Atherosclerosis 2019, 282, 19-28. [CrossRef]

4. Yusuf, S.; Hawken, S.; Ounpuu, S.; Dans, T.; Avezum, A.; Lanas, F.; McQueen, M.; Budaj, A.; Pais, P.; Varigos, J.; et al. Effect of potentially modifiable risk factors associated with myocardial infarction in 52 countries (the INTERHEART study): Case-control study. Lancet 2004, 364, 937-952. [CrossRef]

5. Ma, D.; Sakai, H.; Wakabayashi, C.; Kwon, J.-S.; Lee, Y.; Liu, S.; Wan, Q.; Sasao, K.; Ito, K.; Nishihara, K.; et al. The prevalence and risk factor control associated with noncommunicable diseases in China, Japan, and Korea. J. Epidemiol. 2017, 27, 568-573. [CrossRef]

6. Lynch, S.V.; Pedersen, O. The Human Intestinal Microbiome in Health and Disease. N. Engl. J. Med. 2016, 375, 2369-2379. [CrossRef]

7. Woting, A.; Blaut, M. The Intestinal Microbiota in Metabolic Disease. Nutrients 2016, 8, 202. [CrossRef]

8. Qin, J.; Li, Y.; Cai, Z.; Li, S.; Zhu, J.; Zhang, F.; Liang, S.; Zhang, W.; Guan, Y.; Shen, D.; et al. A metagenome-wide association study of gut microbiota in type 2 diabetes. Nature 2012, 490, 55-60. [CrossRef]

9. Tong, X.; Xu, J.; Lian, F.; Yu, X.; Zhao, Y.; Xu, L.; Zhang, M.; Zhao, X.; Shen, J.; Wu, S.; et al. Structural Alteration of Gut Microbiota during the Amelioration of Human Type 2 Diabetes with Hyperlipidemia by Metformin and a Traditional Chinese Herbal Formula: A Multicenter, Randomized, Open Label Clinical Trial. mBio 2018, 9, e02392-17. [CrossRef]

10. Yan, Q.; Gu, Y.; Li, X.; Yang, W.; Jia, L.; Chen, C.; Han, X.; Huang, Y.; Zhao, L.; Li, P.; et al. Alterations of the Gut Microbiome in Hypertension. Front. Cell Infect. Microbiol 2017, 7, 381. [CrossRef]

11. Nishijima, S.; Suda, W.; Oshima, K.; Kim, S.-W.; Hirose, Y.; Morita, H.; Hattori, M. The gut microbiome of healthy Japanese and its microbial and functional uniqueness. DNA Res. 2016, 23, 125-133. [CrossRef] [PubMed] 
12. Hashimoto, Y.; Hamaguchi, M.; Kaji, A.; Sakai, R.; Osaka, T.; Inoue, R.; Kashiwagi, S.; Mizushima, K.; Uchiyama, K.; Takagi, T.; et al. Intake of sucrose affects gut dysbiosis in patients with type 2 diabetes. J. Diabetes Investig. 2020. [CrossRef]

13. Naito, Y.; Takagi, T.; Inoue, R.; Kashiwagi, S.; Mizushima, K.; Tsuchiya, S.; Itoh, Y.; Okuda, K.; Tsujimoto, Y.; Adachi, A.; et al. Gut microbiota differences in elderly subjects between rural city Kyotango and urban city Kyoto: An age-gender-matched study. J. Clin. Biochem. Nutr. 2019, 65, 125-131. [CrossRef] [PubMed]

14. Takagi, T.; Naito, Y.; Inoue, R.; Kashiwagi, S.; Uchiyama, K.; Mizushima, K.; Tsuchiya, S.; Dohi, O.; Yoshida, N.; Kamada, K.; et al. Differences in gut microbiota associated with age, sex, and stool consistency in healthy Japanese subjects. J. Gastroenterol. 2019, 54, 53-63. [CrossRef]

15. Takagi, T.; Naito, Y.; Inoue, R.; Kashiwagi, S.; Uchiyama, K.; Mizushima, K.; Tsuchiya, S.; Okayama, T.; Dohi, O.; Yoshida, N.; et al. The influence of long-term use of proton pump inhibitors on the gut microbiota: An age-sex-matched case-control study. J. Clin. Biochem. Nutr. 2018, 62, 100-105. [CrossRef]

16. Li, W.; Fu, L.; Niu, B.; Wu, S.; Wooley, J. Ultrafast clustering algorithms for metagenomic sequence analysis. Brief. Bioinform. 2012, 13, 656-668. [CrossRef]

17. Caporaso, J.G.; Kuczynski, J.; Stombaugh, J.; Bittinger, K.; Bushman, F.D.; Costello, E.K.; Fierer, N.; Peña, A.G.; Goodrich, J.K.; Gordon, J.I.; et al. QIIME allows analysis of high-throughput community sequencing data. Nat. Methods 2010, 7, 335-336. [CrossRef]

18. Wang, Q.; Garrity, G.M.; Tiedje, J.M.; Cole, J.R. Naive Bayesian classifier for rapid assignment of rRNA sequences into the new bacterial taxonomy. Appl. Env. Microbiol. 2007, 73, 5261-5267. [CrossRef]

19. DeSantis, T.Z.; Hugenholtz, P.; Larsen, N.; Rojas, M.; Brodie, E.L.; Keller, K.; Huber, T.; Dalevi, D.; Hu, P.; Andersen, G.L. Greengenes, a chimera-checked 16S rRNA gene database and workbench compatible with ARB. Appl. Env. Microbiol. 2006, 72, 5069-5072. [CrossRef]

20. Langille, M.G.; Zaneveld, J.; Caporaso, J.G.; McDonald, D.; Knights, D.; Reyes, J.A.; Clemente, J.C.; Burkepile, D.E.; Thurber, R.L.V.; Knight, R.; et al. Predictive functional profiling of microbial communities using 16S rRNA marker gene sequences. Nat. Biotechnol. 2013, 31, 814-821. [CrossRef]

21. Kanehisa, M.; Goto, S.; Sato, Y.; Kawashima, M.; Furumichi, M.; Tanabe, M. Data, information, knowledge and principle: Back to metabolism in KEGG. Nucleic Acids Res. 2014, 42, D199-D205. [CrossRef] [PubMed]

22. Adachi, K.; Sugiyama, T.; Yamaguchi, Y.; Tamura, Y.; Izawa, S.; Hijikata, Y.; Ebi, M.; Funaki, Y.; Ogasawara, N.; Goto, C.; et al. Gut microbiota disorders cause type 2 diabetes mellitus and homeostatic disturbances in gut-related metabolism in Japanese subjects. J. Clin. Biochem. Nutr. 2019, 64, 231-238. [CrossRef] [PubMed]

23. Lahti, L.; Salonen, A.; Kekkonen, R.A.; Salojärvi, J.; Jalanka-Tuovinen, J.; Palva, A.; Orešič, M.; de Vos, W.M. Associations between the human intestinal microbiota, Lactobacillus rhamnosus GG and serum lipids indicated by integrated analysis of high-throughput profiling data. PeerJ 2013, 1, e32. [CrossRef] [PubMed]

24. Nirmalkar, K.; Murugesan, S.; Pizano-Zárate, M.L.; Villalobos-Flores, L.E.; García-González, C.; Morales-Hernández, R.M.; Nuñez-Hernández, J.A.; Hernández-Quiroz, F.; Romero-Figueroa, M.D.S.; Hernández-Guerrero, C.; et al. Gut Microbiota and Endothelial Dysfunction Markers in Obese Mexican Children and Adolescents. Nutrients 2018, 10, 2009. [CrossRef] [PubMed]

25. Lambeth, S.M.; Carson, T.; Lowe, J.; Ramaraj, T.; Leff, J.W.; Luo, L.; Bell, C.J.; Shah, V.O. Composition, Diversity and Abundance of Gut Microbiome in Prediabetes and Type 2 Diabetes. J. Diabetes Obes. 2015, 2 , 1-7. [CrossRef]

26. Gomez-Arango, L.F.; Barrett, H.L.; Wilkinson, S.A.; Callaway, L.K.; McIntyre, H.D.; Morrison, M.; Nitert, M.D. Low dietary fiber intake increases Collinsella abundance in the gut microbiota of overweight and obese pregnant women. Gut Microbes 2018, 9, 189-201. [CrossRef]

27. Frost, F.; Storck, L.J.; Kacprowski, T.; Gärtner, S.; Rühlemann, M.; Bang, C.; Franke, A.; Völker, U.; Aghdassi, A.A.; Steveling, A.; et al. A structured weight loss program increases gut microbiota phylogenetic diversity and reduces levels of Collinsella in obese type 2 diabetics: A pilot study. PLoS ONE 2019, 14, e0219489. [CrossRef]

28. Tong, A.J.; Hu, R.K.; Wu, L.X.; Lv, X.-C.; Li, X.; Zhao, L.-N.; Liu, B. Ganoderma polysaccharide and chitosan synergistically ameliorate lipid metabolic disorders and modulate gut microbiota composition in high fat diet-fed golden hamsters. J. Food Biochem. 2020, 44, e13109. [CrossRef]

29. Wan, X.; Li, T.; Liu, D.; Chen, Y.; Liu, Y.; Liu, B.; Zhang, H.; Zhao, C. Effect of Marine Microalga Chlorella pyrenoidosa Ethanol Extract on Lipid Metabolism and Gut Microbiota Composition in High-Fat Diet-Fed Rats. Mar. Drugs 2018, 16, 498. [CrossRef] 
30. Thingholm, L.B.; Rühlemann, M.C.; Koch, M.; Fuqua, B.; Laucke, G.; Boehm, R.; Bang, C.; Franzosa, E.A.; Hübenthal, M.; Rahnavard, A.; et al. Obese Individuals with and without Type 2 Diabetes Show Different Gut Microbial Functional Capacity and Composition. Cell Host Microbe 2019, 26, 252-264. e210. [CrossRef]

31. Li, H.; Liu, B.; Song, J.; An, Z.; Zeng, X.; Li, J.; Jiang, J.; Xie, L.; Wu, W. Characteristics of Gut Microbiota in Patients with Hypertension and/or Hyperlipidemia: A Cross-Sectional Study on Rural Residents in Xinxiang County, Henan Province. Microorganisms 2019, 7, 399. [CrossRef] [PubMed]

(C) 2020 by the authors. Licensee MDPI, Basel, Switzerland. This article is an open access article distributed under the terms and conditions of the Creative Commons Attribution (CC BY) license (http://creativecommons.org/licenses/by/4.0/). 